\title{
Lidar Data Inversion for Cirrus Clouds: An Approach Based on a Statistical Analysis of In Situ Microphysical Measurements
}

\author{
GUY FEBVRE \\ LaMP, CNRS, Université Blaise Pascal, Clermont-Ferrand, France
}

(Manuscript received 17 February 1993, in final form 4 February 1994)

ABSTRACT

\begin{abstract}
The problem of the lidar equation inversion lies in the fact that it requires a lidar calibration or else a reference value from the studied medium. This paper presents an approach to calibrate the lidar by calculating the constant $A k$ (lidar constant $A$ multiplied by the ratio of backscatter coefficient to extinction coefficient $k$ ). This approach is based on statistical analysis of in situ measurements. This analysis demonstrates that the extinction coefficient has a typical probability distribution in cirrus clouds. The property of this distribution, as far as the attenuation of laser beam in the cloud, is used as a constraint to calculate the value of $A k$. The validity of this method is discussed and results compared with two other inversion methods.
\end{abstract}

\section{Introduction}

Cirrus clouds have recently been recognized to have a great influence on weather and climate through their effect on the radiative energy budget in the atmosphere (Liou 1986). To achieve a better understanding of global climate change, a good description of the optical properties of these clouds is necessary. The lidar instrument is particularly adapted to study the optical properties of transparent or semitransparent clouds. Nevertheless, the inversion of the lidar equation is not trivial. On the one hand, it requires either the calibration of the lidar or a reference value of the studied atmosphere. On the other hand, we assume that the backscatter coefficient and the extinction coefficient can be linked according to a power law.

From the Klett's algorithm (Klett 1981), which is based on a logarithm solution of the lidar equation, Fergusson et al. (1983) and Mulders (1984) determined the reference value of the extinction coefficient using molecular scattering. Their approach is a very useful technique. Nevertheless a calibration is sometimes preferable when it is difficult to estimate a reference value of the atmosphere. For example, a calibration based on the strong extinction of cumulus cloud and the invariant value of the backscatter to extinction ratio in water clouds has been developed by Elouragini (1991). For optically thin clouds like cirrus, a new concept has been developed by Ruppersberg et al. $(1990,1992)$. This method, called "the shadow calibration," uses the laser beam attenuation through multilayered clouds. However, these methods are not

Corresponding author address: Guy Febvre, LaMP, 24 Av. des Landais, Aubiere 63177, France. always appropriate for a systematic study of cirrus clouds.

In this paper, we propose a new technique of lidar calibration. This method is developed for cirrus study from a vertically pointing $1.06-\mu \mathrm{m}$-wavelength lidar. The sensitivity of this lidar does not allow the detection of the molecular backscatter, so none of the methods based on molecular backscatter can be used.

In our method we demonstrate with in situ measurements that the integrated probability density function (IPDF) of the extinction coefficient has an exponential form. With a cloud model, we show the effect of the laser attenuation through the cloud on the IPDF shape. Application to one cloud case demonstrates how to implement the method. A comparison with two other methods presents the advantages and the limits of this new approach.

The data used in this study were collected during the International Cirrus Experiment (ICE), which was carried out to support the International Satellite Cloud Climatology Project. This experiment took place in the North Sea during fall 1989 (Raschke et al. 1990).

\section{Presentation of the extinction coefficient distribution}

\section{a. In situ measurements}

The in situ measurements come from a PMS 2D-C particle spectrometer probe (Knollenberg 1976) mounted on the Merlin aircraft operated by the Centre d'Aviation Météorologique of Météo-France. The method provides a size spectrum distributed over 30 channels, each with a $25-\mu \mathrm{m}$ resolution $(25-750-\mu \mathrm{m}$ size range), at $1-\mathrm{Hz}$ frequency. The primary sizing parameter used for all particle images is the mean di- 
ameter. This diameter is the average of the image dimensions measured parallel and perpendicular to the orientation of the photodiode array. The method of data processing and accuracies have been discussed in detail in previous papers (Gayet et al. 1993a).

The extinction coefficient can be calculated from the size distribution by (Platt 1973)

$$
\sigma=\sum_{i=1}^{i=30} \pi Q_{e} N_{i}(a) a_{i}^{2},
$$

with $N_{i}$ the particle number of radius $a_{i}, i$ the class number, and $Q_{e}$ the extinction efficiency equal to 2 in the large-particle approximation (radius greater than $20 \mu \mathrm{m}$ for considered lidar wavelength $1.06 \mu \mathrm{m}$ ).

\section{b. Extinction coefficient distribution}

Figure 1 gives an example of microphysical data of a cirrus cloud sampled at $5900 \mathrm{~m}$ above sea level $\left(-20.6^{\circ} \mathrm{C}\right)$ on 16 October 1989 . This figure shows a time series of the mean particle size, as well as total concentration and extinction coefficient calculated with (1).

The integral of the probability density function (IPDF) of the extinction coefficient is calculated according to these data. Figure 2 shows the IPDF in a semilog representation for the cloud portrayed on Fig. 1. The solid line represents the corresponding linear regression. The correlation coefficient of 0.99 suggests that the linear function is a representative fit of the extinction coefficient IPDF in a semilog graph.

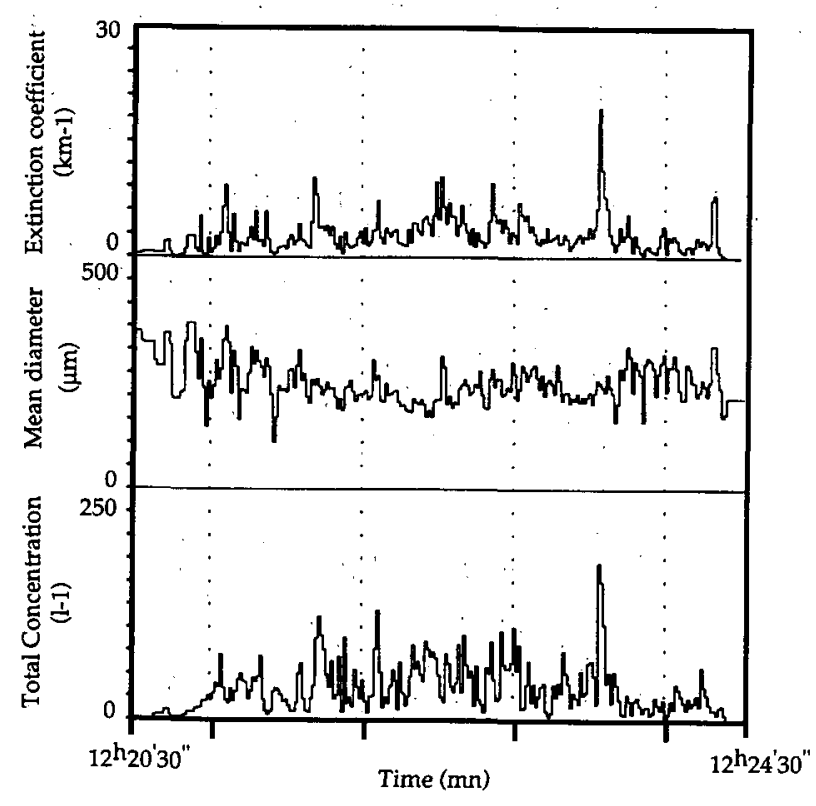

FIG. 1. Time series of microphysical parameters measured in cirrus at $6-\mathrm{km}$ altitude between 1220:30 and 1224:30 UTC 16 October 1989.

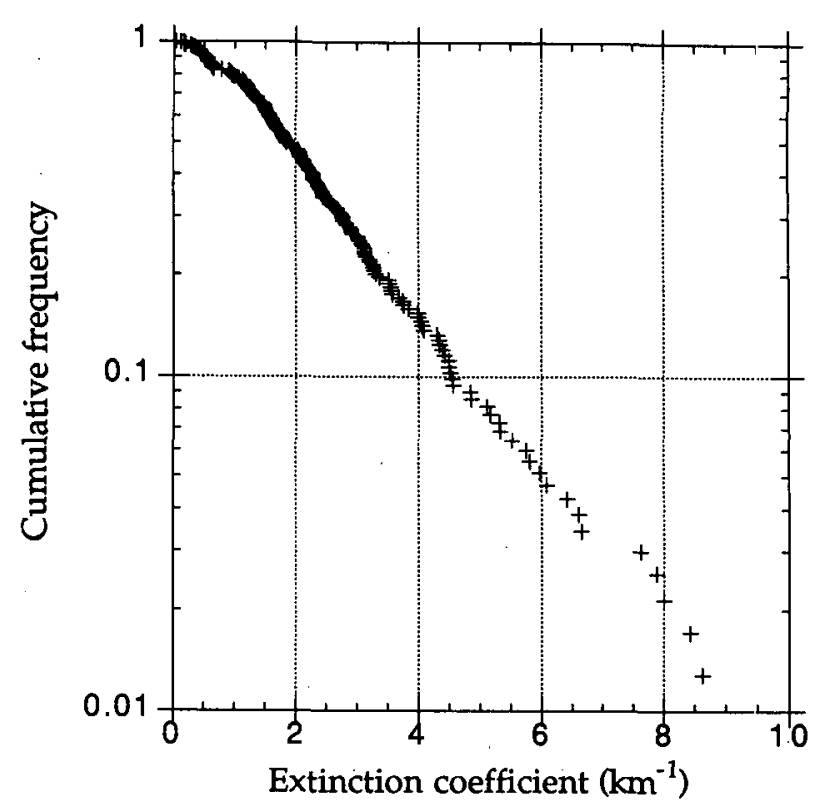

FIG. 2. Cumulative frequency of extinction coefficient obtained from the data presented in Fig. 1.

To check the exponential behavior of the extinction coefficient IPDF, all cirrus sampled by the Merlin aircraft during ICE were analyzed. These data correspond to seven flights, which are divided into 59 cloud sequences.

The extinction coefficient IPDF is calculated for each cloud sequence, and a linear regression is applied: In Table 1, we report the corresponding correlation coefficient and suitable microphysical parameters of the sampled cirrus. Figure 3, representing the histogram of the correlation coefficient, shows that $75 \%$ of the studied cases present a correlation coefficient greater than 0.97. As suggested by Lopez (1977), we first investigated the possibility that the probability density function (PDF) would be described by a lognormal distribution, but the data revealed that an exponential IPDF is much more accurate. Therefore, we assume that the exponential relationship represents statistically the extinction coefficient IPDF in cirrus.

\section{c. Discussion}

\section{1) ACCURACIES OF IN SITU MEASUREMENTS}

It should be noted that the Merlin aircraft was not equipped with a PMS 2D-P probe for the measurement of the larger ice crystals. This may introduce only small error in particle sizing because no ice particles around $1 \mathrm{~mm}$ were sampled during the present study. Much larger uncertainties probably come from the lack of reliable measurements of small ice particles (typically from 3 to $50 \mu \mathrm{m}$ ). Tentative estimates have been done either in extrapolating measured PMS 1D-C size spectra (Stephens 1980) or by using FSSP measurements 
TABLE 1. Characteristics of cloud sequences obtained in cirrus during ICE. They are reported as the date, the time of the sequences, the cloud length, the flight altitude $(\mathrm{km})$ and the corresponding temperature $\left({ }^{\circ} \mathrm{C}\right)$, the mean particle diameter $(\mu \mathrm{m})$, and the correlation coefficient of the IPDF.

\begin{tabular}{|c|c|c|c|c|c|c|c|}
\hline Date & Number & $\begin{array}{c}\text { Time } \\
\text { (UTC) }\end{array}$ & $\begin{array}{c}\text { Cloud length } \\
(\mathrm{km})\end{array}$ & $\begin{array}{c}\text { Flight level } \\
(\mathrm{km})\end{array}$ & $\begin{array}{c}\text { Temperature } \\
\left({ }^{\circ} \mathrm{C}\right)\end{array}$ & $\begin{array}{c}\text { Mean } \\
\text { diameter } \\
(\mu \mathrm{m})\end{array}$ & Correlation coefficient \\
\hline 15 September 1989 & $\begin{array}{l}1 \\
2 \\
3 \\
4 \\
5 \\
6 \\
7 \\
8\end{array}$ & $\begin{array}{l}1043: 30 \\
1050: 30 \\
1053: 30 \\
1109: 45 \\
1115: 15 \\
1150: 00 \\
1153: 15 \\
1155: 30\end{array}$ & $\begin{array}{r}9.2 \\
19.9 \\
105.3 \\
29.8 \\
67.0 \\
7.5 \\
17.0 \\
18.9\end{array}$ & $\begin{array}{l}5.7 \\
6.7 \\
7.6 \\
7.9 \\
7.9 \\
7.9 \\
7.9 \\
7.9\end{array}$ & $\begin{array}{l}-13.7 \\
-19.3 \\
-26.2 \\
-29.6 \\
-29.6 \\
-30.4 \\
-30.4 \\
-30.5\end{array}$ & $\begin{array}{r}98 \\
82 \\
94 \\
109 \\
99 \\
66 \\
105 \\
125\end{array}$ & $\begin{array}{l}0.98 \\
0.90 \\
0.96 \\
0.98 \\
0.99 \\
0.94 \\
0.96 \\
0.98\end{array}$ \\
\hline 20 September 1989 & $\begin{array}{l}1 \\
2 \\
3 \\
4 \\
5 \\
6 \\
7 \\
8 \\
9\end{array}$ & $\begin{array}{l}1111: 00 \\
1142: 00 \\
1207: 00 \\
1210: 00 \\
1212: 30 \\
1248: 00 \\
1251: 30 \\
1301: 00 \\
1302: 00\end{array}$ & $\begin{array}{r}20.8 \\
24.4 \\
13.3 \\
9.4 \\
13.2 \\
9.6 \\
33.2 \\
7.4 \\
10.9\end{array}$ & $\begin{array}{l}6.5 \\
6.6 \\
6.8 \\
6.8 \\
6.8 \\
7.4 \\
7.4 \\
7.4 \\
7.4\end{array}$ & $\begin{array}{l}-16.3 \\
-17.2 \\
-18.7 \\
-18.5 \\
-18.2 \\
-22.8 \\
-22.9 \\
-22.9 \\
-22.9\end{array}$ & $\begin{array}{r}92 \\
101 \\
171 \\
106 \\
72 \\
80 \\
52 \\
45 \\
54\end{array}$ & $\begin{array}{l}0.98 \\
0.99 \\
0.98 \\
0.98 \\
0.98 \\
0.94 \\
0.98 \\
0.97 \\
0.99\end{array}$ \\
\hline 9 October 1989 & $\begin{array}{l}1 \\
2\end{array}$ & $\begin{array}{l}\text { 0804:45 } \\
\text { 0806:00 }\end{array}$ & $\begin{array}{r}9.7 \\
23.4\end{array}$ & $\begin{array}{l}6.7 \\
6.7\end{array}$ & $\begin{array}{l}-27.2 \\
-27.4\end{array}$ & $\begin{array}{r}94 \\
100\end{array}$ & $\begin{array}{l}0.95 \\
0.95\end{array}$ \\
\hline 10 October 1989 & $\begin{array}{r}1 \\
2 \\
3 \\
4 \\
5 \\
6 \\
7 \\
8 \\
9 \\
10 \\
11 \\
12\end{array}$ & $\begin{array}{l}\text { 1328:00 } \\
1356: 00 \\
1401: 00 \\
1405: 00 \\
1406: 15 \\
1410: 15 \\
1411: 15 \\
1421: 00 \\
1423: 30 \\
1525: 30 \\
1527: 30 \\
1530: 00\end{array}$ & $\begin{array}{r}154.8 \\
30.7 \\
26.9 \\
8.4 \\
10.2 \\
5.8 \\
9.7 \\
15.5 \\
54.2 \\
5.9 \\
19.8 \\
123.6\end{array}$ & $\begin{array}{l}6.7 \\
6.7 \\
6.8 \\
7.1 \\
7.3 \\
7.3 \\
7.3 \\
7.3 \\
7.1 \\
6.7 \\
6.7 \\
6.7\end{array}$ & $\begin{array}{l}-26.9 \\
-27.2 \\
-27.7 \\
-29.7 \\
-31.3 \\
-31.6 \\
-31.6 \\
-31.7 \\
-30.3 \\
-26.9 \\
-27.1 \\
-27.2\end{array}$ & $\begin{array}{r}103 \\
121 \\
123 \\
94 \\
144 \\
47 \\
134 \\
136 \\
83 \\
68 \\
103 \\
109\end{array}$ & $\begin{array}{l}0.99 \\
0.97 \\
0.99 \\
0.96 \\
0.96 \\
0.97 \\
0.86 \\
0.91 \\
0.97 \\
0.98 \\
0.97 \\
0.99\end{array}$ \\
\hline 12 October 1989 & $\begin{array}{r}1 \\
2 \\
3 \\
4 \\
5 \\
6 \\
7 \\
8 \\
9 \\
10 \\
11 \\
12 \\
13\end{array}$ & $\begin{array}{l}0949: 15 \\
0955: 30 \\
1000: 00 \\
1002: 15 \\
1004: 45 \\
1011: 45 \\
1017: 30 \\
1022: 30 \\
1052: 30 \\
1109: 30 \\
1137: 45 \\
1141: 00 \\
1144: 00\end{array}$ & $\begin{array}{l}23.4 \\
31.4 \\
17.5 \\
15.8 \\
36.8 \\
15.5 \\
39.3 \\
46.8 \\
39.3 \\
46.4 \\
24.2 \\
21.8 \\
11.7\end{array}$ & $\begin{array}{l}6.7 \\
6.7 \\
6.7 \\
6.7 \\
6.7 \\
6.7 \\
6.7 \\
6.7 \\
6.7 \\
6.3 \\
6.4 \\
6.4 \\
6.9\end{array}$ & $\begin{array}{l}-25.6 \\
-25.8 \\
-26.3 \\
-26.3 \\
-26.5 \\
-26.6 \\
-26.5 \\
-26.3 \\
-26.4 \\
-23.3 \\
-23.4 \\
-23.4 \\
-27.1\end{array}$ & $\begin{array}{r}134 \\
99 \\
113 \\
85 \\
81 \\
69 \\
123 \\
116 \\
138 \\
120 \\
116 \\
126 \\
106\end{array}$ & $\begin{array}{l}0.97 \\
0.98 \\
0.99 \\
0.97 \\
0.99 \\
0.97 \\
0.99 \\
0.95 \\
0.99 \\
0.99 \\
0.99 \\
0.96 \\
0.96\end{array}$ \\
\hline 13 October 1989 & $\begin{array}{l}1 \\
2 \\
3 \\
4 \\
5\end{array}$ & $\begin{array}{l}1124: 20 \\
1141: 45 \\
1208: 00 \\
1214: 00 \\
1241: 00\end{array}$ & $\begin{array}{r}5.1 \\
38.4 \\
9.4 \\
14.8 \\
7.6\end{array}$ & $\begin{array}{l}7.3 \\
7.3 \\
7.3 \\
7.3 \\
7.7\end{array}$ & $\begin{array}{l}-32.1 \\
-30.3 \\
-30.8 \\
-30.4 \\
-33.8\end{array}$ & $\begin{array}{r}34 \\
106 \\
59 \\
120 \\
33\end{array}$ & $\begin{array}{l}0.98 \\
0.99 \\
0.97 \\
0.99 \\
0.98\end{array}$ \\
\hline 16 October 1989 & $\begin{array}{r}1 \\
2 \\
3 \\
4 \\
5 \\
6 \\
7 \\
8 \\
9 \\
10\end{array}$ & $\begin{array}{l}0948: 45 \\
0951: 15 \\
0959: 30 \\
1008: 00 \\
1010: 00 \\
1038: 30 \\
1153: 00 \\
1159: 30 \\
1220: 30 \\
1230: 15\end{array}$ & $\begin{array}{l}15 \\
35.9 \\
64.3 \\
15.2 \\
47.2 \\
30.7 \\
34.8 \\
26.1 \\
34.8 \\
52.1\end{array}$ & $\begin{array}{l}6.7 \\
6.8 \\
6.8 \\
6.8 \\
6.8 \\
7.4 \\
7.7 \\
7.2 \\
5.9 \\
6.8\end{array}$ & $\begin{array}{l}-26.1 \\
-26.6 \\
-26.2 \\
-25.9 \\
-26.0 \\
-31.3 \\
-32.8 \\
-29.9 \\
-20.6 \\
-25.8\end{array}$ & $\begin{array}{r}126 \\
104 \\
109 \\
110 \\
112 \\
147 \\
74 \\
103 \\
122 \\
110\end{array}$ & $\begin{array}{l}0.99 \\
0.99 \\
0.95 \\
0.97 \\
0.99 \\
0.99 \\
0.98 \\
0.99 \\
0.99 \\
0.99\end{array}$ \\
\hline
\end{tabular}




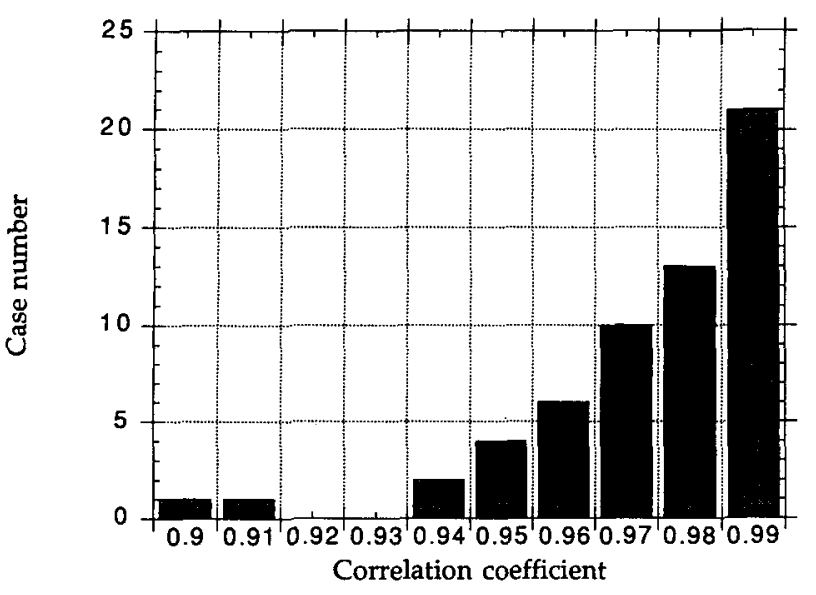

Fug. 3. Histogram of the correlation coefficient of the best linear fit of the extinction coefficient cumulative frequency. These data come from the 59 studied cases reported in Table 1.

(Platt et al. 1989). Comparisons between FSSP (forward-scattering spectrometer probe) and 2D-C measurements made on the Merlin aircraft confirm that the FSSP is not a reliable probe for the measurement of ice particles with a complex shape [as already pointed out by Gardiner and Hallet (1985)]. Consequently, no measurement of small particles is considered in the systematic data processing. Therefore, large particles compared to the lidar wavelength are assumed, and the calculation of the extinction coefficient from (1) can assign a constant value to the extinction efficiency $Q_{e}$. This equation is not applicable when the particle radius is lower than $20 \mu \mathrm{m}$.

The absolute values of the extinction coefficient must be considered carefully because of the large uncertainties on the concentration measurement. These errors are due to the difficult estimation of the probe sampling area and detection response (Gayet and Soulage 1992). Nevertheless, the relative fluctuations of this parameter reflect the actual proportional changes in cloud microphysical characteristics. The linear characteristic of IPDF in a semilog representation can be considered, but the slope and the intercept of the linear fit must be used carefully.

The correlation of the IPDF linear fit must be used cautiously when the mean diameter is small. Indeed the uncertainties on the concentration measurement of small particles may be large and the hypothesis of $Q_{e}$ constant is not always reliable.

\section{2) Correlation COEFFicient}

In this study, the IPDF is plotted in a semilog graph. The linear behavior is isolated by a best linear fit. Because of this representation, the correlation coefficient naturally presents a high value. The linearity is significant only for a correlation greater than 0.97 .

\section{3) CLOUD MORPHOLOGY}

The data sampling must have at least one hundred points for the statistical analysis to be correct. That is to say, the clouds like young cloud cells or contrails, with little spatial extent, are not very well represented. Consequently, the values of the correlation coefficient of the IPDF must be used carefully.

\section{d. Conclusion}

In conclusion, the extinction coefficient IPDF presents an exponential law in $75 \%$ of the studied cases of cirrus during the ICE experiment. The exponential relationship can be considered as a good representation of the extinction coefficient IPDF. However, clciuds that are small or have particles with small mean diameter do not lend themselves to this method.

\section{Application to lidar equation inversion}

\section{a. Equation and inversion}

From the scattering theory, the backscatter power $P$ at the altitude $z$ is linked to the emitted laser energy by

$$
P(z)=E_{0} \frac{A}{z^{2}} \beta_{r}(z) \tau^{2}(z),
$$

where $A$ is the lidar constant, $\beta_{r}$ the backscatter coefficient, $E_{0}$ the emitted energy laser, and $\tau$ the transmission of the medium. Here, $P$ and $z$ represent the lidar measurements, where $z$ is the range between the backscattered pulse and the receiver.

Transmission $\tau$ is defined as

$$
\tau^{2}=\exp \left[-2 \int_{z_{0}}^{z} \sigma_{e}\left(z^{\prime}\right) d z^{\prime}\right] .
$$

The true backscatter coefficient can be calculated from (2), if the lidar constant $A$ and the attenuation $\tau$ are known. To reduce the numbers of unknowns, the extinction coefficient is considered proportional to the backscatter coefficient

$$
\beta_{r}(z)=k \sigma_{e}(z) .
$$

The lidar equation becomes

$$
P(z)=E_{0} \frac{A k}{z^{2}} \sigma_{e}(z) \tau^{2}(z) .
$$

In ice cloud, the phase function that governs the value of $k$ varies with crystal habit, orientation, and particle sizes. Sassen (1978) gave values of $k\left(4 \pi \beta_{r} / \sigma_{e}\right.$ in his terminology) measured by an $\mathrm{He}-\mathrm{Ne}$ laser in laboratory. His values ranged from 0.26 for ice crystal to 0.41 for aggregates. Platt et al. $(1987,1989)$ gave some values of $k$ as function of the temperature. For clouds warmer than $-40^{\circ} \mathrm{C}$, the value of $k$ is usually constant $\left(0.013 \mathrm{sr}^{-1}\right)$, but a greater value $\left(0.032 \mathrm{sr}^{-1}\right)$ 
has been determined at $-62^{\circ} \mathrm{C}$. These results show that large uncertainties remain on the determination of $k$ in cirrus clouds. Consequently, $k$ is considered unknown but assumed to have a constant value within each cloud investigated.

To determine the extinction coefficient from (5), the values of $A$ multiplied by $k(A k)$ must be known.

Another way is the use of Klett's formalism (Klett 1981). The differential lidar equation is

$$
\frac{d S}{d z}=\frac{1}{\beta_{r}} \frac{d \beta_{r}}{d z}-2 \sigma_{e}
$$

where

$$
S(z)=\ln \left[P(z) z^{2}\right]
$$

and the stable solution is

$$
\sigma_{e}(z)=\frac{\exp \left[S(z)-S\left(z_{t}\right)\right]}{\frac{1}{\sigma_{e}\left(z_{t}\right)}+2 \int_{z}^{z_{t}} \exp \left[S\left(z^{\prime}\right)-S\left(z_{t}\right)\right] d z^{\prime}},
$$

where $z_{t}$ represents the altitude of the cloud top. This solution does not require any lidar calibration. However, a reference value of the extinction coefficient and the value of $k$ must be determined.

A number of methods have been developed to determine these variables to invert the lidar equation. The simplest one uses the molecular backscatter from both sides of the cloud to calculate the cloud optical thickness. This method associated with the Klett algorithm gives good results. Another way is the calculation of the optical depth with another instrument, for instance a radiometer.

The next paragraphs describe how the exponential distribution of the extinction coefficient is used to determine the unknowns of the lidar equation.

\section{b. Specific properties of the extinction coefficient distribution}

We have seen in section $3 a$ that the lidar measures the attenuated backscatter coefficient, and that the extinction coefficient (not attenuated) IPDF has an exponential form as seen from the in situ measurements. This section shows the extinction coefficient distribution is sensitive to attenuation of the laser in the cloud. These characteristics can be shown with simulations of lidar measurements.

A cloud is simulated assuming the extinction coefficient IPDF follows the same exponential relationship at all levels in the cloud. Figure 4 represents the exponential relationship obtained from the in situ measurements shown on Fig. 1. The cloud is defined as a grid of 200 horizontal points on 50 levels. The distance between two horizontal points is equal to the distance between two lidar pulses $(100 \mathrm{~m})$. The distance between two vertical points is equal to $10 \mathrm{~m}$, which is similar to the lidar vertical resolution $(6 \mathrm{~m})$. Two hundred values of extinction coefficients are used from the data reported in Fig. 4. These values are set on all points of one level with a random process and this level is duplicated throughout the cloud. The model leads to a cloud characterized by a horizontal heterogeneity, only because of the constant value of the extinction coefficient along a specific vertical.

The attenuated backscatter coefficient is calculated with the lidar equation on all grid points. The corresponding IPDFs are obtained on three levels: base, 50, and $100 \mathrm{~m}$ above the cloud base (Fig. 5). This figure shows that the IPDF follows an exponential relationship at the cloud base, where the attenuation effect is low. On the contrary, no exponential form is observed at higher levels in the cloud. It should be noticed that the choice of the parameter value used in this model (intercept and slope parameters of the IPDF, horizontal, and vertical resolution ) can affect the absolute values of the results portrayed in Fig. 5, but do not affect the relative variations of the curves between the base and the other levels.

Consequently, our results suggest that the IPDF of the attenuated backscatter coefficient is sensitive to the attenuation effect. Indeed, upward-pointed lidar backscatter measurements follow an exponential distribution at the cloud base, but not at the top, although the true extinction coefficient distribution is exponential throughout the cloud.

Our inversion method proposes to reproduce this exponential distribution of the extinction coefficient in determining the value of $A k$.

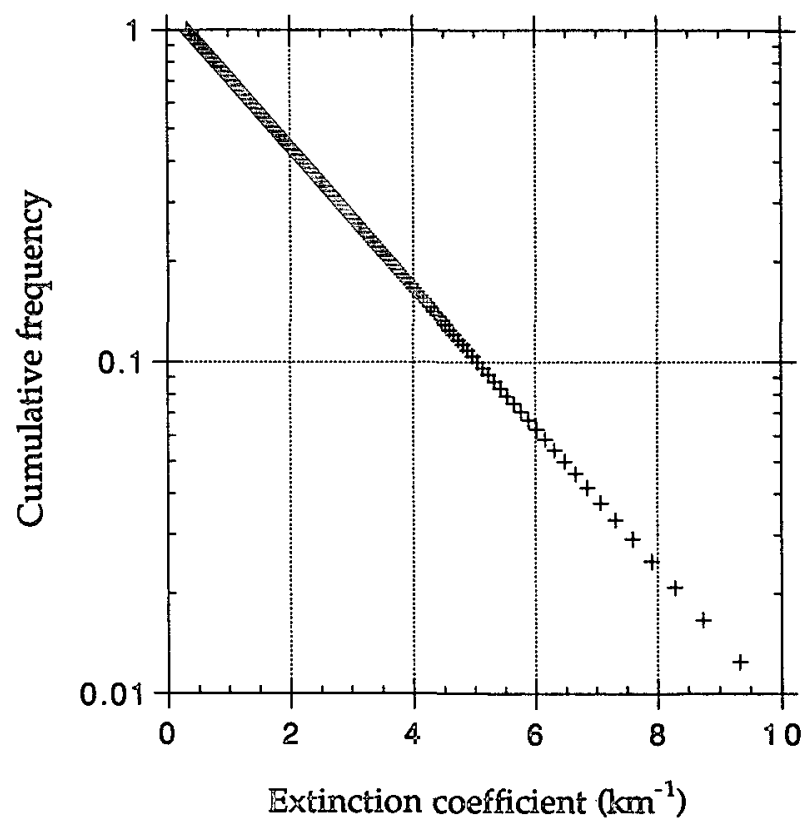

FIG. 4. Extinction coefficient cumulative frequency used in the cloud model. The equation is $F_{C}=A \exp (-B \sigma)$. The constants $A$ $=1.16807$ and $B=-0.4864$ come from the case in Fig. 2 . 


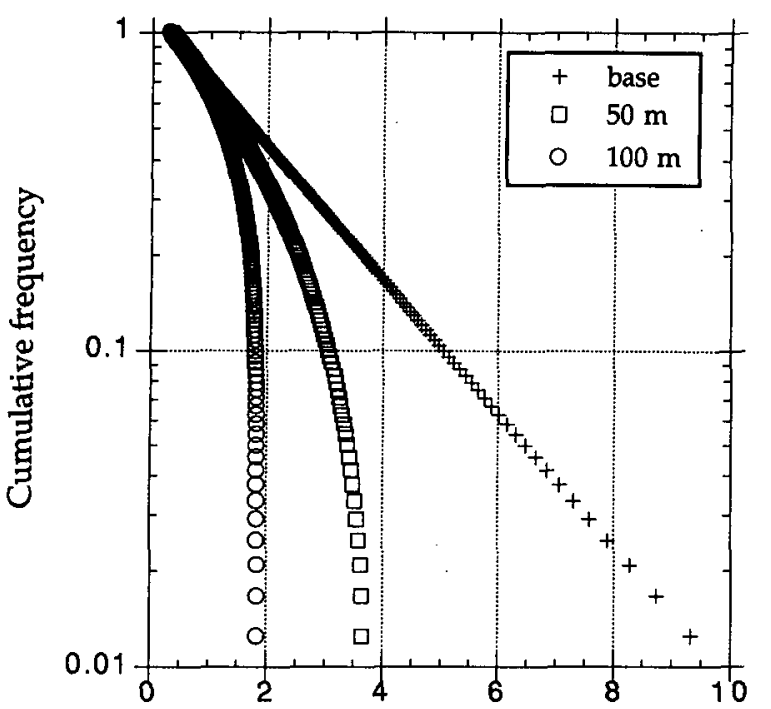

Attenuated backscatter coefficient (arbitrary units)

FIG. 5. Cumulative frequency of the attenuated backscatter coefficient for three levels in the simulated cloud.

\section{c. Inversion method}

To solve the lidar equation, the constant $A k$ must be known. The determination of this value requires an additional constraint as shown in section 3a. This constraint is obtained considering the exponential behavior of the IPDF. The correct value of the constant $A k$ gives the correct attenuation correction, which in turn must lead to the extinction coefficient IPDF observed with in situ measurements.

The following procedure is applied to determine this constant.

(i) The extinction coefficient is calculated in all points of the cloud for a given value of the constant $A k$.

(ii) At the chosen level, the IPDF is calculated and a linear regression on these data performed to obtain the corresponding correlation coefficient.

(iii) Steps (i) and (ii) are repeated with different values of $A k$, in order to obtain the correlation coefficient as a function of the constant $A k$. The maximum of this curve gives the value of $A k$ that best fits the constraint.

The chosen level must be in the upper part of the cloud so that the attenuation effect of the laser beam can be measured. In the next section we present an application of this method. The value of $A k$ is determined at two levels in the cloud: the top and the middle.

\section{d. Application}

The lidar measurements came from the ALEX-F lidar mounted on the Do228 aircraft operating at $1.06 \mu \mathrm{m}$. Each upward pulse had a vertical resolution equal to
TABLE 2. Characteristics of the ALEX-F lidar mounted on the Do228 aircraft of the DLR.

\begin{tabular}{ll}
\hline \hline Laser type & UTG 72 international laser systems \\
Wavelength & $1.06 \mu \mathrm{m}$ \\
Energy per shot & $400 \mathrm{~mJ}$ \\
Beam quality & multimode \\
Beam divergence & $2 \mathrm{mrad}$ \\
Shot frequency & Single shot up to $1 \mathrm{~Hz}$ \\
Telescope type & Cassegrain \\
Primary mirror & $35 \mathrm{~cm}$ \\
Focus & $400 \mathrm{~cm}$ \\
Field of view & $3 \mathrm{mrad}$ \\
Receiver & RCA 4517 photomultiplier and \\
Digitizer & YAG 444 photodiode \\
\hline
\end{tabular}

$6 \mathrm{~m}$. The main characteristics of the lidar are summarized in Table 2 (Moerl et al. 1981). Figure 6 represents the attenuated backscatter coefficient cross section. This cloud is related to the cirrus case study carried out on 13 October 1989 during the intensive field observation of ICE. The meteorological situation was characterized by a cold front passing over the experiment area at midday. The examination of Fig. 6 shows that the cirrus cloud top is around $8500 \mathrm{~m}\left(-37^{\circ} \mathrm{C}\right)$ and the cloud base is around $6600 \mathrm{~m}\left(-20^{\circ} \mathrm{C}\right)$. The lidar measurements between 1123:00 and 1124:30 UTC will be considered later on. This cloud case has been chosen because it is the subject of another study concerning the microphysical and optical differences between natural cirrus and contrail (Gayet et al. 1993b).

Before doing the inversion procedure, it is necessary to check that the attenuated backscatter coefficient distribution is exponential at the cloud base, as we assumed in section 3b. In Fig. 7, the IPDF is represented in a semilog graph for three different levels inside the cloud layer. The IPDF follows a quasi-exponential relationship at the cloud base, but not at higher levels. These results are in good agreement with those simu-

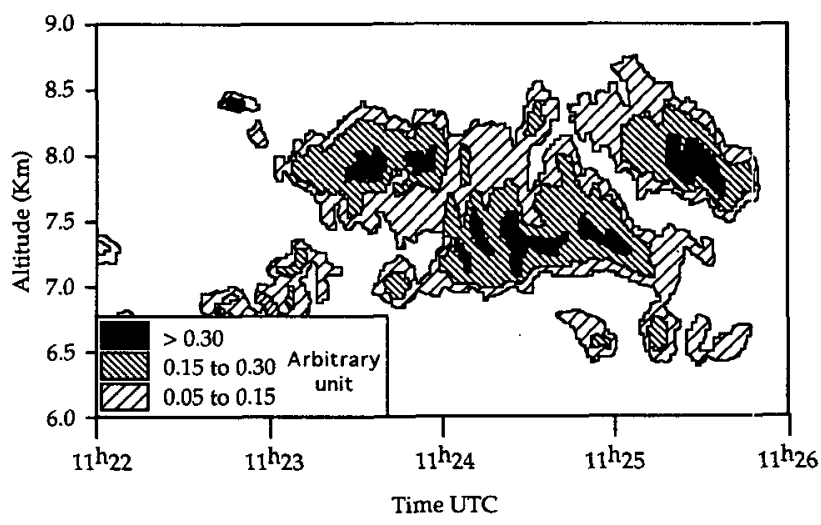

FIG. 6. Vertical cross section of the attenuated backscatter coefficient from the Do228/ALEX-F lidar between 1122:00 and 1126:00 UTC 13 October 1989. 


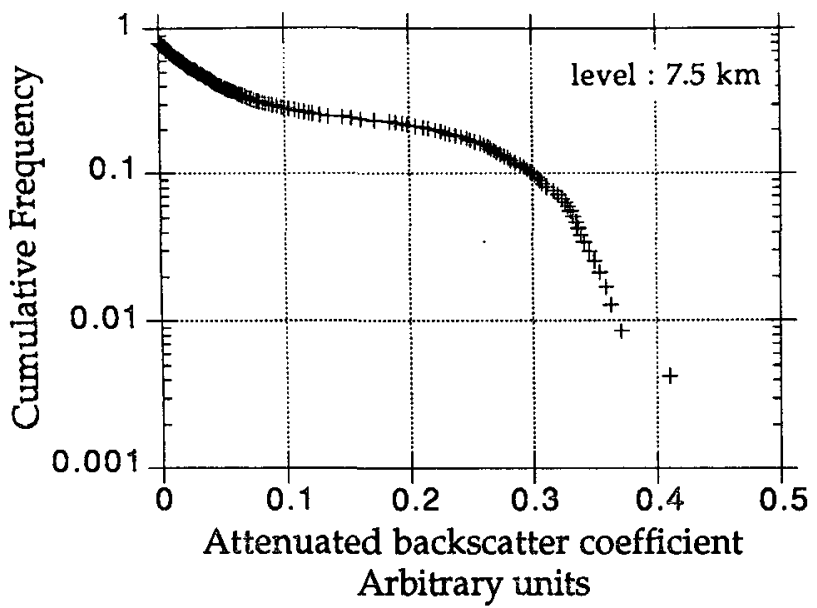

those of the simulated IPDFs because of the vertical heterogeneity of natural cloud.

To determine the value of $A k$, we consider the hypothesis that the extinction coefficient IPDF follows an exponential law that is assumed valid for the whole cloud. Figure 8a shows constant $A k$ versus the correlation coefficient of the IPDF best linear fit, obtained with the data of the $8.0-\mathrm{km}$ altitude. The maximum of this curve corresponds to a constant $A k$ equal to 0.50 . The same calculation is made at the cloud middle $(7.4 \mathrm{~km})$. In Fig. $8 \mathrm{~b}$, the result shows a value of $A k$ equal to 0.42 . Both of these values are used to compute the extinction coefficient on the whole cloud, as shown in Figs. 9a and 9b.
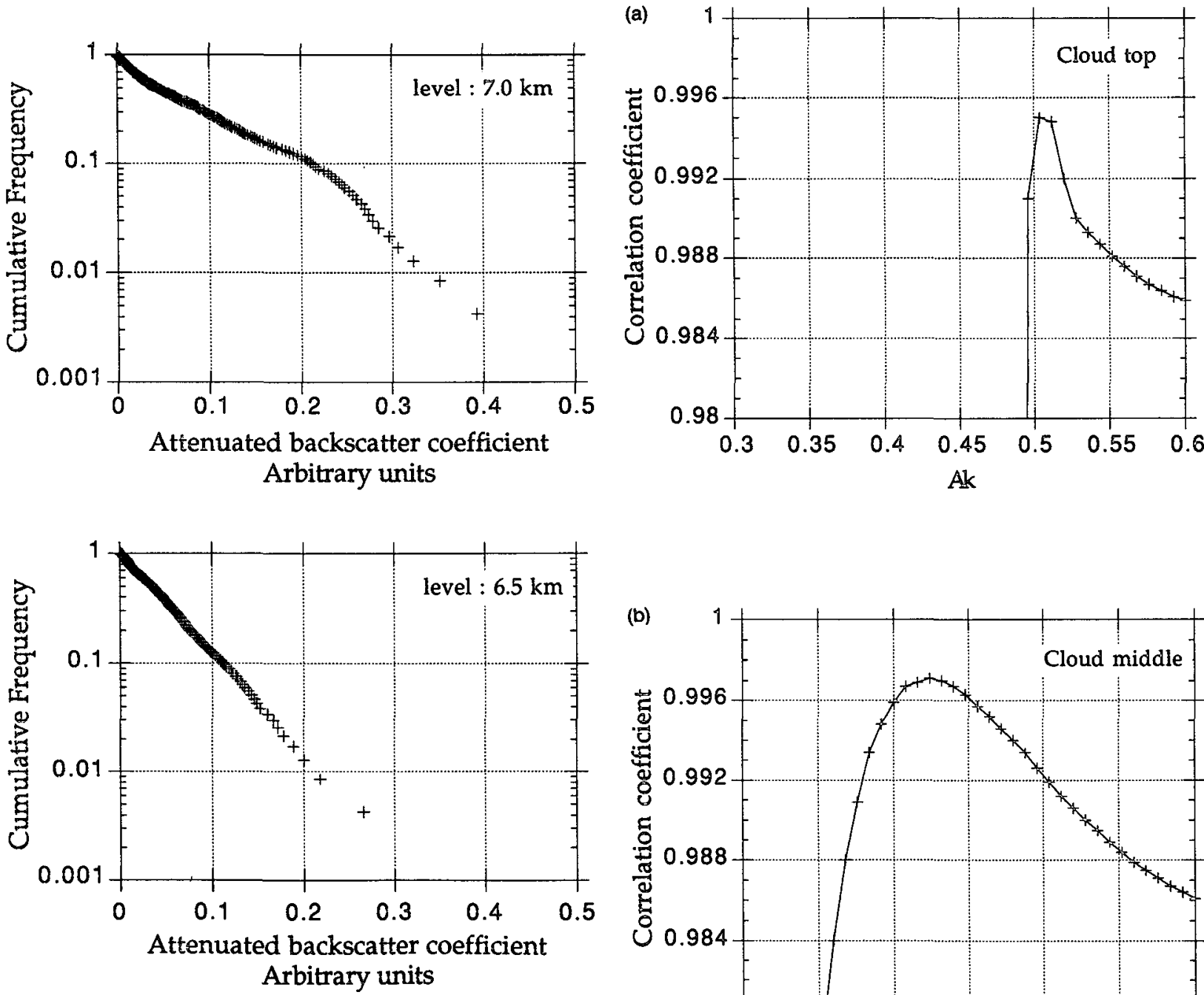

FIG. 7. Cumulative frequency of the attenuated backscatter coefficient for three levels in the cirrus cloud presented in Fig. 6.

lated in the model discussed in section $3 \mathrm{~b}$. The relative positions of the experimental IPDF curves between the cloud base and the cloud top are quite different from

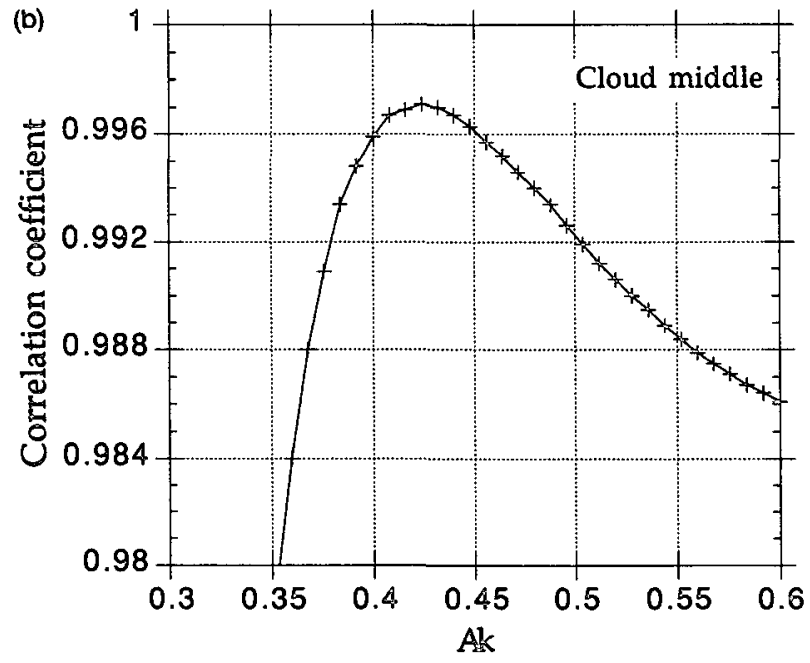

FIG. 8. Correlation coefficient of the best linear fit calculated from the extinction coefficient cumulative frequency as function of the constant $A k$ : (a) top level, (b) middle level. 

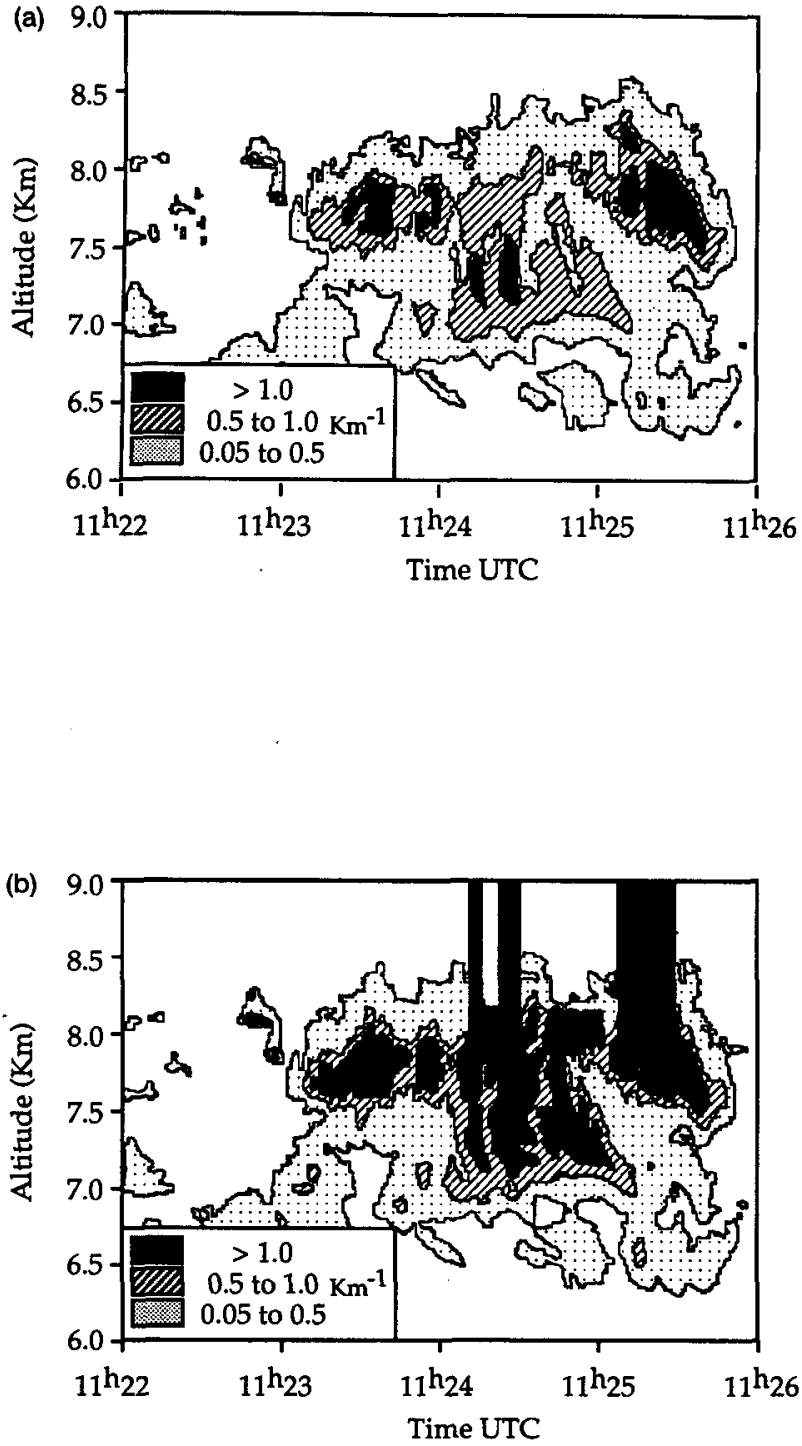

Fig. 9. As in Fig. 6 in terms of extinction coefficient calculated from the constants $A k$ determined in Fig. 8: (a) $A k=0.50$, (b) $A k=0.42$.

\section{e. Comparison}

Klett's algorithm associated with PRT5 measurements and the "shadow calibration" are used to compare the results of our calibration. These chosen methods do not use the aerosol backscatter. Furthermore, they have been developed for the same lidar, so the calibration constants can be directly compared.

\section{1) KLETT'S METHOD}

As shown in section 3a, Klett's method is based on the Bernouilli solution of the differential lidar equation (6). The necessary reference value is estimated from a PRT5 radiometer (Gratzki 1989). The simultaneous radiometer measurement allows one to calculate the absorption optical thickness in the infrared $\delta_{A}^{\mathrm{IR}}$ from the cloud emissivity $\epsilon$

$$
\delta_{A}^{\mathrm{IR}}=-\ln (1-\epsilon) .
$$

For a given cloud particle distribution, the Mie theory links the optical thickness in the IR with the optical thickness in the lidar wavelength:

$$
\delta_{\lambda}=\alpha \delta_{A}^{\mathrm{IR}},
$$

where $\alpha$ is a proportional coefficient that is approximately equal to two in the range of cloud particles. This optical thickness calculated by the IR measurements is used to calculate the reference value in the Klett's method by the equation

$$
\beta_{e}\left(z_{t}\right)=\frac{\exp \left[S\left(z_{t}\right)\right]\left[1-\exp \left(-2 \alpha \delta_{A}^{\mathrm{IR}}\right)\right]}{\exp \left(-2 \alpha \delta_{A}^{\mathrm{IR}}\right) 2 \int_{z_{0}}^{z_{t}} \exp \left[S\left(z^{\prime}\right)\right] d z^{\prime}},
$$

where $z_{t}$ is the altitude of the cloud top and $z_{0}$ the altitude of the cloud base. The calculation of the extinction coefficient is made with the lidar measurements $[P(z)$ and $z]$ and from the PRT5 measurement $(\epsilon)$.

\section{2) SHADOW CALIBRATION}

This method (Ruppersberg et al. 1990, 1992) is based on the shading of a laser beam hitting upward upon a cirrus cloud (labeled "ci" in equations hereafter) through a lower-lying cloud (labeled "llc"). The lidar is calibrated by the shading.

The method consists in calculating the true cirrus signal in the shadowed region by determination of $A k$. The hypothesis made is that the true cirrus signal has the same behavior in the shadowed region as in the nonshadowed region.

The unknown $(A k)$ is determined by solving the equation

$$
\sum_{x_{0}}^{x_{1}}\left[\frac{E\left(x, \Delta z_{\mathrm{ci}}\right)}{\tau^{2}\left(x, \Delta z_{\mathrm{llc}}\right)}-E_{\mathrm{INT}}\left(x, \Delta z_{\mathrm{ci}}\right)\right]=0,
$$

where $E$ is the sum of the relative lidar counts at the position $x$ and $\Delta z$ the geometrical depth of the considered cloud. The first term in the bracket corresponds to the cirrus shadowed by the contrail and the second term to the nonshadowed cirrus, where $\tau$ is the shadowing cloud transmission.

The authors give the calibration constant $A k$ with a $20 \%$ accuracy. This method can be used with the Klett's formalism or with a classical iterative method for the transmission calculation. As the lidar measurement is the only one required for the determination of the extinction coefficient of a multilayered cloud, the method is particularly interesting. 


\section{f. Results}

Klett's method is applied to the sequence described in Fig. 6. Figure 10 shows the emissivity calculated from PRT5 measurement as well as the deduced optical thickness at $1.06 \mu \mathrm{m}$ versus time.

Figures 11 show the optical thickness calculated with the "PRT5 Klett's algorithm" versus the optical thickness obtained with our proposed method, first with $A k$ equal to 0.50 and second with $A k$ equal to 0.42 . The linear regression in the first case gives a slope equal to 0.70 with a correlation coefficient equal to 0.96 . In the second case slope equals 1.16 and correlation coefficient equals 0.93 . The high values of these coefficients and the slope close to 1 show that the two methods are in good agreement.

The shadow calibration has been made on the sequence displayed on Fig. 6 between 1123:00 and 1124:30 UTC. The shadowing region is located between 1123:00 and 1124:00 UTC and between 5.2 and $7.4 \mathrm{~km}$. The constant $A k$ found with this method is equal to 0.42 . This value is equal to the one found by our method in the cloud middle (Ruppersberg et al. 1990).

The author of the shadow calibration analysis indicates that this value of $A k$ leads to inadmissibly large values of the extinction coefficient $\left(\sigma_{e}>400 \mathrm{~km}^{-1}\right)$ in the upper parts of the cloud. This problem of instability is discussed in section 4.

These two comparisons show that the better calibration is $A k$ equal to 0.42 , even if some points present aberrant values of extinction coefficient.

\section{Discussion}

Our method is based on the following four hypotheses.

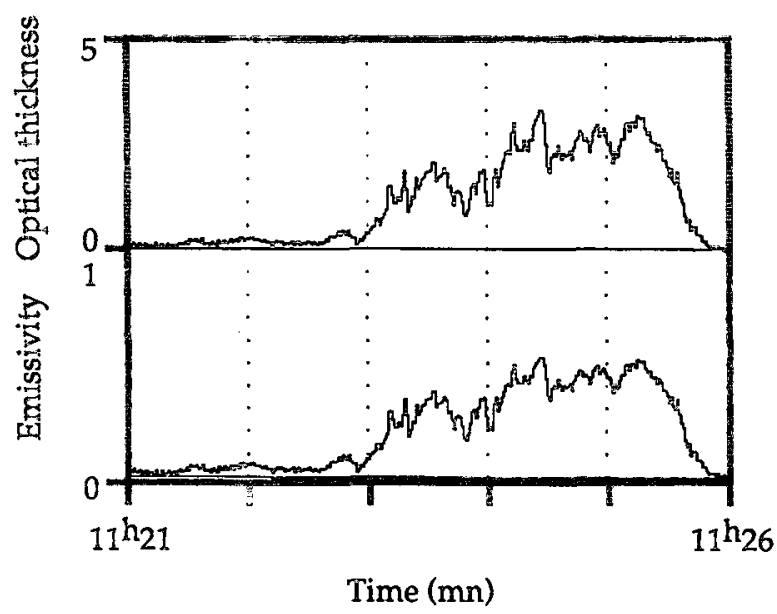

FIG. 10. Time series of the emissivity and corresponding visible optical thickness between 1121:00 and 1126:00 UTC 13 October 1989.
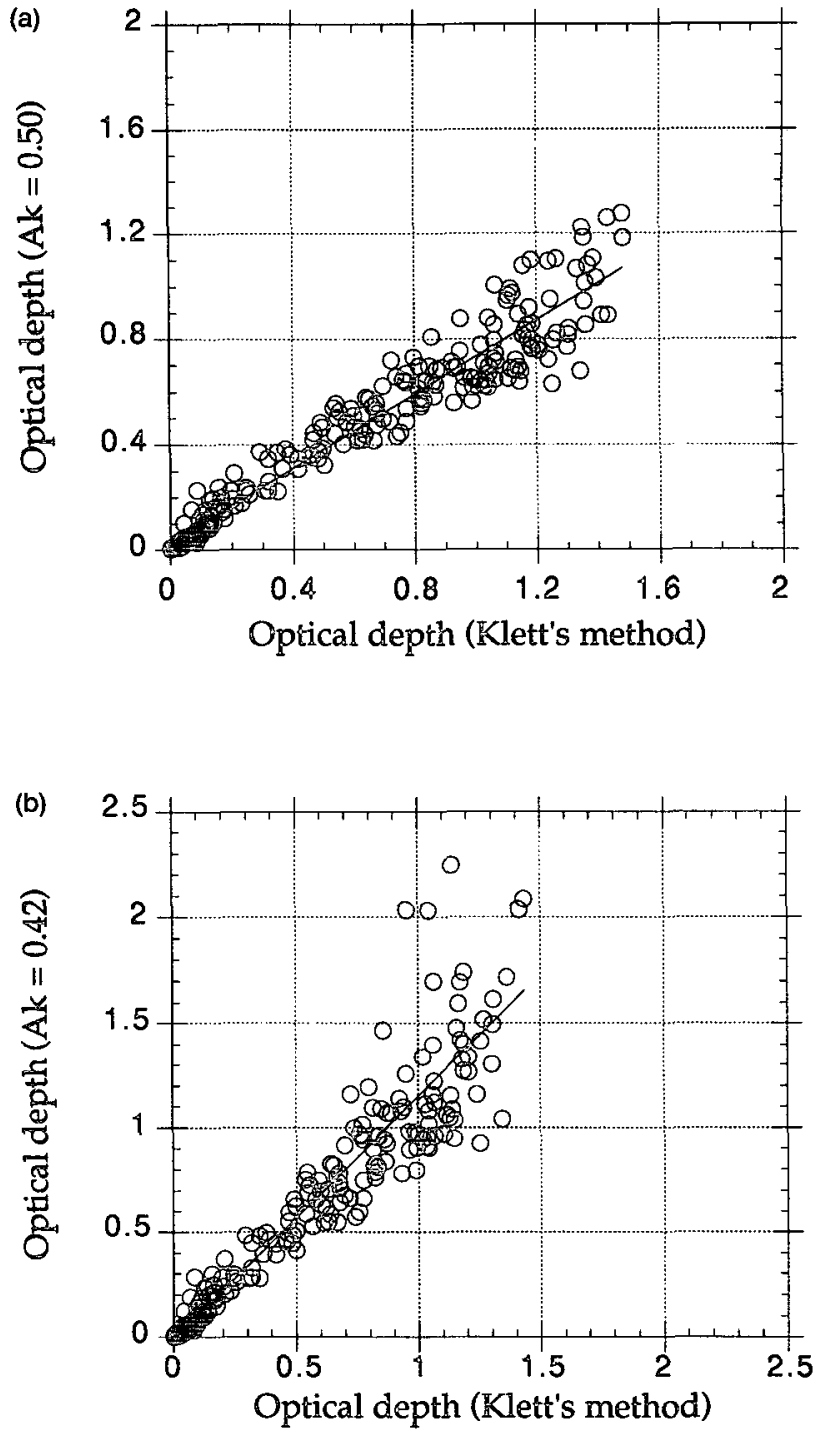

FIG. 11. Comparison of the optical thickness calculated with our $A k$ values and with Klett's method. The data come from the sequence presented in Fig. 9: (a) $A k=0.50$, (b) $A k=0.42$.

(i) The extinction coefficient IPDF follows an exponential relationship, shown with in situ measurements. Up to now, we have no physical interpretation. However, we may recall that only $75 \%$ of the studied cases during the $\mathbb{C} C E$ present such a feature.

(ii) The extinction coefficient $\mathrm{PPDF}$ is sensitive to the laser attenuation in the cloud. The depth of the cloud must be sufficient for this phenomenon to be observable. Practically, an optical depth greater than 0.2 is necessary.

(iii) The value $A k$ is assumed constant in the studied cloud. Moreover the statistical analysis requires a sampling of at least one hundred lidar pulses. The minimum distance to calculate $A k$ in a cirrus $(7 \mathrm{~km}$ with an aircraft speed equal to $70 \mathrm{~m} \mathrm{~s}^{-1}$ and one lidar pulse 
per second) is too large for a representative study of the variation of $k$.

(iv) The multiple scattering factor $\eta$ is neglected ( $\eta$ $=1$ ) because the introduction of factor $\eta$ in the lidar equation increases the number of unknowns. It appears in the lidar equation as a power $(<1)$ of the transmission. The consequence is that if multiple scattering is assumed equal to 1 , the measured extinction coefficient is greater than the actual extinction coefficient. Platt (1980) showed that for cirrus clouds the parameter $\eta$ varies from about 0.42 to 1 according to the depth of cloud penetration, the cloud optical depth, and the cloud extinction coefficient. It also varies according to the distance between cloud and lidar and the laser receiver aperture. If the hypothesis $\eta=1$ is used, extinction coefficient values are overestimated. Hence, we observe unstable behavior of the method when optical thickness is high. To treat the problem of inadmissible large values of the extinction coefficient (observed with optical thickness larger than 1.5), it is necessary to use multiple scattering value lower than 1 . It is not a good solution to calculate $A k$ in cloud top even if it reduces very high values. Therefore, in our approach, the better value of $A k$ is that determined in the middle level. As $\eta$ is equal to 1 , the inversion requires caution that large values of the optical depth can have large errors.

To limit the errors due to the two previous points, we can estimate the dispersion of the $k$ and $\eta$ values by plotting the emissivity $\epsilon$ deduced from the PRT5 measurements versus the integrated received lidar power $\gamma^{\prime}$ (Platt et al. 1989). These two parameters can be expressed by (Platt et al. 1989)

$$
\gamma^{\prime}=\frac{A k}{2 \eta}\{1-\exp [2 \eta \alpha \ln (1-\epsilon)]\},
$$

where $\alpha$ is the ratio of visible extinction coefficient to IR absorption coefficient and $\epsilon$ is the emissivity.

If the corresponding plot of $\gamma^{\prime}$ against $\epsilon$ is fitted by this equation, the dispersion of the plot represented by the error bars gives information on the cloud heterogeneity and the variation of the suitable parameters. Figure 12 presents this processing for the sequence of the studied cloud (Gayet et al. 1993b). The results show that the dispersion is lower than $10 \%$. We can reasonably deduce that the parameters $k$ and $\eta$ do not vary much.

The verification of the cloud homogeneity is necessary to eliminate some possible zones where $k$ presents anomalous values, for example, contrail (Gayet et al. 1993b), specular reflection from oriented crystals (Rockwitz 1989), and others.

This paper presents an iterative method of lidar equation inversion by using the extinction coefficient IPDF. The principle can be used to invert the lidar equation with the Klett algorithm. The constraint (extinction coefficient IPDF is exponential) can be used to determine the reference value. Likewise, another

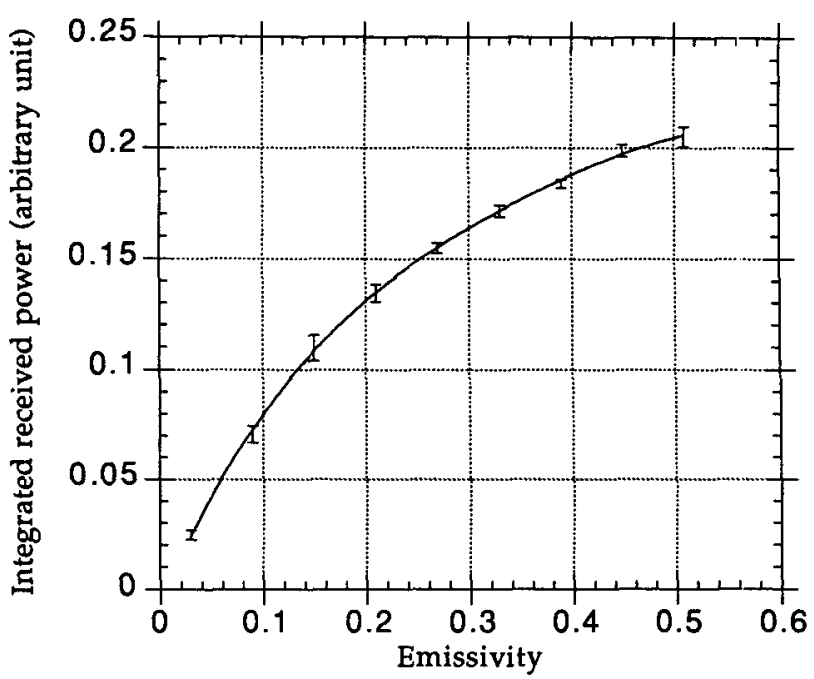

FIG. 12. Integrated received lidar power vs emissivity for the data from the cirrus cloud presented in Fig. 6.

statistical constraint [e.g., the lognormal probability distribution (Lopez 1977)] can be used if it is justified by in situ measurements and if it is sensitive to the laser beam attenuation in cloud.

As a conclusion, this paper presents a new approach to calibrate a lidar. The technique uses a typical statistical behavior of the extinction coefficient, which is sensitive to the laser beam attenuation. This behavior is used as a constraint in order to solve the lidar equation. The proposed technique gives. results in good agreement with two comparative methods. Nevertheless larger use requires the following:

(i) a study of the introduction of the multiple-scattering factor in the lidar equation, to make possible the study of optically thick clouds;

(ii) a study to reduce the horizontal size of the sampling, in order to estimate the variation of the parameter $k$.

The main advantage is that only lidar measurements are used, and the originality is that the cloud is considered as a statistical sample.

Acknowledgments. This work was supported by grants from CNRS/PNEDC No. 88/50/N/5080, DRET/Université Blaise Pascal No. 891183 , and EPOCH/EUCREX-0002-c (MB). I would like to thank P. Wendling and G. H. Ruppersbergs of DLR, Oberpfaffenhofen, Germany. Thanks are also due to J. F. Gayet for his helpful comments.

\section{REFERENCES}

Elouragini, S., 1991: Etude des propriétés optiques et géométriques des cirrus par télédétection optique active (lidar) et passive (radiométrie). Ph.D. thesis, Université Paris VI, $279 \mathrm{pp}$.

Fergusson, J. A., and D. H. Stephens, 1983: Algorithm for inverting lidar returns. Appl. Opt., 22, 3673-3675. 
Gardiner, B. A., and J. Hallet, 1985: Degradation of in-cloud droplet measurements in the presence of ice particle. J. Atmos. Oceanic Technol., 2, 171-180.

Gayet, J.-F., and R. G. Soulage, 1992: Observation of high ice particle concentrations in convective cells and cloud glaciation evolution. Quart. J. Roy. Meteor. Soc., 118, 177-190.

- P. A. Brown, and F. Albers, 1993a: A comparison of in-cloud measurements obtained with six PMS 2D-C probes. J. Atmos. Oceanic Technol., 10, 180-194.

- , G. Febvre, G. Brogniez, and P. Wendling, 1993b: Microphysical and optical properties of cirrus and contrails; cloud field study on 13 October 1989. Proc. Sixth ICE/EUCREX Workshop, Stockholm, 30-39.

Gratzki, A., 1989: Bestimmung der vertikalen Verteilung des Eisgehaltes in Cirruswolken aus kombinierten Lidar und InfrarotRadiometer Messungen. Ph.D. thesis, University of Köln, 100 pp.

Klett, J. D., 1981: Stable analytical inversion solution for processing lidar returns. Appl. Opt., 22, 211-220.

Knollenberg, R. G., 1976: Three new instruments for cloud physics measurements: The 2D spectrometer, the forward scattering spectrometer probe and the active scattering aerosol spectrometer. Preprints, Seventh Int. Cloud Physics Conf., Boulder, CO, Amer. Meteor. Soc., 554-561.

Liou, K. N., 1986: Influence of cirrus cloud on weather and climate processes: A global perspective. Mon. Wea. Rev., 14, 11671199.

Lopez, R. E., 1977: Some properties of convective plume and small fair-weather cumulus fields as measured by acoustic and lidar sounders. J. Appl. Meteor., 16, 861-865.

Moerl, P., M. E. Reinhardt, W. Renger, X. Schellhase, 1981: The use of the airborne lidar system "ALEX-F" for aerosol tracing in the lower troposphere. Beitr. Phys. Atmos., 45, 403-410.
Mulders, J. M., 1984: Algorithm for inverting lidar returns: Comment. Appl. Opt., 23, 2855-2856.

Platt, C. M. R., 1973: Lidar and radiometric observations of cirrus clouds. J. Atmos. Sci., 30, 1191-1204.

- 1980: Remote sounding of high clouds. III: Monte Carlo calculations of the multiple-scattered lidar return. J. Atmos. Sci., $38,156-167$.

—, and A. C. Dilley, 1981: Remote sounding of high clouds. IV: Observed temperature variations in cirrus optical properties. $J$. Atmos. Sci., 38, 1069-1082.

- J. D. Spinhirne, and W. D. Hart, 1989: Optical and microphysical properties of a cold cirrus cloud: Evidence for regions of small particles. J. Geophys. Res., 94, 11 151-11 164.

- J. C. Scott, and A. C. Dilley, 1987: Remote sounding of high clouds. Part VI: Optical properties of midlatitude and tropical cirrus. J. Atmos. Sci., 44, 729-747.

Raschke, E., J. Schmetz, J. Heintzenberg, R. Kandel, and R. Saunders, 1990: The International Cirrus Experiment (ICE). A joint European effort. ESA $J$., 臬4, 193-199.

Rockwitz, K. D., 1989: Scattering properties of horizontally oriented ice crystal columns in cirrus clouds. Part 1. Appl. Opt., 28, 41034110.

Ruppersberg, G. H., and W. Renger, 1990: Shadow technique for improved inversion of lidar data to cirrus optical depth. Proc. Third ICE/EUCREX Workshop, Villeneuve d'Ascq, 57-59.

- , and 1992: Shadow technique for improved inversion of lidar data to cirrus optical depth. European Space Agency Report, ESA-TT, 1263 pp.

Sassen, K., 1978: Backscattering cross sections for hydrometeors measurements at 6328 angstrom. Appl. Opt., 直7, 804-806.

Stephens, G. L., 1980: Radiative properties of cirrus clouds in the infrared region. J. Atmos. Sci., 37, 435-446. 